\title{
Thermoelectric performance of lanthanum telluride produced via mechanical alloying
}

\author{
Andrew F. May, ${ }^{1}$ Jean-Pierre Fleurial, ${ }^{2}$ and G. Jeffrey Snyder $^{3}$ \\ ${ }^{1}$ Department of Chemical Engineering, California Institute of Technology, 1200 East California Boulevard, \\ Pasadena, California 91125, USA \\ ${ }^{2}$ Jet Propulsion Laboratory, California Institute of Technology, 4800 Oak Grove Drive, Pasadena, California 91109, USA \\ ${ }^{3}$ Department of Materials Science, California Institute of Technology, 1200 East California Boulevard, \\ Pasadena, California 91125, USA
}

(Received 30 April 2008; revised manuscript received 25 June 2008; published 19 September 2008)

\begin{abstract}
Lanthanum telluride $\left(\mathrm{La}_{3-x} \mathrm{Te}_{4}\right)$ has been synthesized via mechanical alloying and characterized for thermoelectric performance. This work confirms prior reports of lanthanum telluride as a good high-temperature thermoelectric material, with $z T \sim 1.1$ obtained at $1275 \mathrm{~K}$. The thermoelectric performance is found to be better than that of SiGe, the current state-of-the-art high-temperature $n$-type thermoelectric material. Inherent selfdoping of the system allows control over carrier concentration via sample stoichiometry. Prior hightemperature syntheses were prone to solute rejection in liquid and vapor phases, which resulted in inhomogeneous chemical composition and carrier concentration. The low-temperature synthesis provides homogeneous samples with acceptable control of the stoichiometry, and thus allows a thorough examination of the transition from a heavily doped degenerate semiconductor to a nondegenerate semiconductor. The effect of carrier concentration on the Hall mobility, Seebeck coefficient, thermal and electrical conductivity, lattice thermal conductivity, and thermoelectric compatibility are examined for $0.03 \leq x \leq 0.33$.
\end{abstract}

DOI: 10.1103/PhysRevB.78.125205

PACS number(s): 73.50.Lw, 72.20.Pa, 74.25.Fy, 84.60.Rb

\section{INTRODUCTION}

\section{A. Motivation}

Thermoelectric devices convert between thermal and electrical fluxes via the solid state and offer significant reliability, compactness, scalability, and quietness. In addition, they are environmentally friendly compared to alternative energy conversion devices, such as compression-based refrigerators. Despite these desirable qualities, thermoelectric devices have low conversion efficiency and are therefore found primarily in niche applications, such as generating power for deepspace science missions, where excellent reliability is paramount. To maximize performance, hot-side temperatures reach $1275 \mathrm{~K}$ and the generator's reliability depends greatly on the thermal stability of the refractory electronics utilized, such as $\mathrm{Si}-\mathrm{Ge}$ alloys.

Lanthanum telluride and other rare-earth chalcogenides of the $\mathrm{Th}_{3} \mathrm{P}_{4}$ structure type have been studied over several decades as potential high-temperature thermoelectric materials, ${ }^{1-5}$ as well as for their superconducting properties. $^{6-8}$ A good summary of the early thermoelectric investigations was provided by Wood, ${ }^{9}$ where lanthanum telluride is identified as the most promising thermoelectric material of the rare-earth chalcogenides examined. This is due to the excellent thermal stability of the rare-earth chalcogenides ${ }^{10}$ and the large dimensionless figure of merit $z T$ of lanthanum telluride. The material's $z T$ characterizes thermoelectric performance and is defined as

$$
z T=\frac{\alpha^{2} \sigma}{\kappa} T,
$$

where $T$ is the absolute temperature, $\alpha$ is the Seebeck coefficient, $\sigma$ is the electrical conductivity, and $\kappa$ is the thermal conductivity. State-of-the-art bulk thermoelectric materials have peak $z T$ values near 1 at the desired operating temperature.

\section{B. Background}

The rare-earth chalcogenide phase of interest exists in the $\mathrm{Th}_{3} \mathrm{P}_{4}$ structure (space group $\bar{I} 43 d$ ), which is shown in Fig. 1 . Tellurium atoms sit on the phosphorus site and experience sixfold coordination with lanthanum via a distorted octahedron. The structure accommodates vacancies on the rareearth site, and up to one-ninth of the lanthanum atoms can be vacant. The presence of vacancies provides disorder and distortion in the lattice, which enhances phonon scattering and

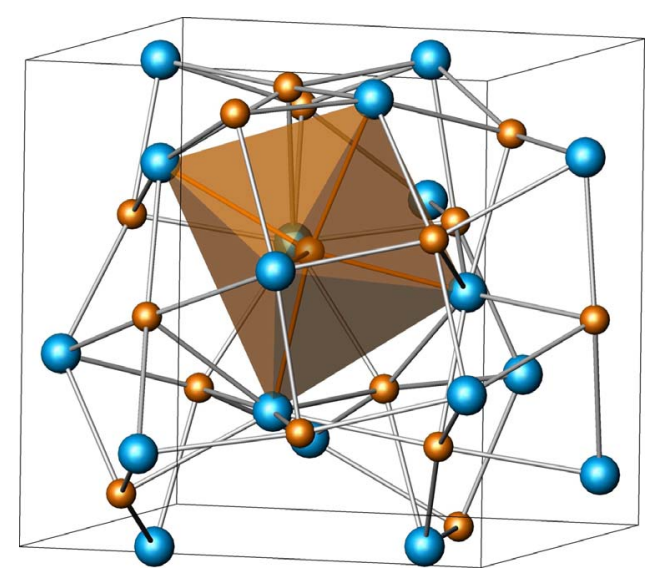

FIG. 1. (Color online) The $\mathrm{La}_{3-x} \mathrm{Te}_{4}$ structure, where the distorted octahedron of lanthanum atoms demonstrates the sixfold lanthanum coordination around tellurium. The structure is shown with lanthanum at full occupancy; however, up to one-ninth of the lanthanum atoms can be vacant. Cyan spheres represent lanthanum atoms and brown spheres represent tellurium atoms. 
contributes to the low lattice thermal conductivity (between approximately 0.4 and $0.8 \mathrm{~W} / \mathrm{m} \mathrm{K}$ ).

The electronic behavior of the rare-earth chalcogenides of interest is easily understood in terms of sample stoichiometry. The composition of interest is described as $\mathrm{La}_{3-x} \mathrm{Te}_{4}$, where $x$ is the number of lanthanum vacancies per formula unit and is restricted to $0 \leq x \leq 1 / 3$. The chemical environment can be considered as $\left(\mathrm{La}^{3+}\right)_{3-x}\left(V_{\mathrm{La}}\right)_{x}\left(\mathrm{Te}^{2-}\right)_{4}\left(e^{1-}\right)_{1-3 x}$, where $V_{\mathrm{La}}$ represents a lanthanum vacancy and $e$ is an electron in the conduction band. The electron concentration is coupled to the lanthanum vacancy concentration, and examination reveals that a metal-insulator transition exists. When the vacancy parameter $x=1 / 3$, the stoichiometry can be written as $\mathrm{La}_{2} \mathrm{Te}_{3}$, and the electrons donated by the $\mathrm{La}^{3+}$ are utilized to complete tellurium reduction, which yields a charge balanced system. For any value of $x<1 / 3$ electrons are introduced into the conduction band and oxidation states are maintained. The carrier concentration of pure $\mathrm{La}_{3-x} \mathrm{Te}_{4}$ is therefore easily calculated in terms of the vacancy parameter and maximum carrier concentration, $n_{\max }=4.5 \times 10^{21} \mathrm{~cm}^{-3}$, by using

$$
n=n_{\max }(1-3 x),
$$

and we define the reduced carrier concentration as $n^{*}$ $=n / n_{\max }$. For small values of $x$, the carrier concentration is large and the transport properties are similar to those of metals or highly degenerate semiconductors. This behavior is maintained for most of the solid solution. It is only when $x$ $\rightarrow 1 / 3$ that the carrier concentration is reduced enough for nondegenerate semiconductor behavior to be observed at moderate temperatures.

Despite extensive investigations in the 1980s, the thermoelectric properties of lanthanum telluride have been difficult to reproducibly confirm for a particular stoichiometry. Previous investigations utilized solid-state diffusion, melt synthesis, or a combination of the two. ${ }^{4,11-13}$ Studies employing melt synthesis required the use of pressure sealed tungsten or tantalum crucibles, and temperatures reached between 2080 and $2280 \mathrm{~K}$. These high-temperature techniques are time consuming and often result in inhomogeneous samples or at a minimum a lack of stoichiometric reproducibility. The high-temperature syntheses are complicated by several factors: (1) vapor phase loss of tellurium (Te melts at $722 \mathrm{~K}$ and boils at $1261 \mathrm{~K})$; (2) high melting temperature of $\mathrm{La}_{3-x} \mathrm{Te}_{4}$ and LaTe $(1992 \mathrm{~K})$; (3) severe sensitivity to oxidation of elemental lanthanum and $\mathrm{La}_{3-x} \mathrm{Te}_{4}$, especially at elevated temperatures; and (4) the presence of a liquid/solid twophase region above the $\mathrm{La}_{3-x} \mathrm{Te}_{4}$ solidus curve, as well as a eutectic reaction involving $\mathrm{La}_{3-x} \mathrm{Te}_{4}$.

The influence of the phase diagram (see Fig. 2) ${ }^{13}$ on melt processing is worth discussing. The presence of a $\mathrm{L}$ $+\mathrm{La}_{3-x} \mathrm{Te}_{4}$ region above the $\mathrm{La}_{3-x} \mathrm{Te}_{4}$ solidus for compositions with greater than approximately 58 at. \% Te causes the composition of the melt and the solid to change upon cooling. In this region, nonequilibrium solidification produces inhomogeneous grains due to the solidification of everchanging compositions of $\mathrm{La}_{3-x} \mathrm{Te}_{4}$, and extensive annealing is required to produce homogenous samples. For samples with less than approximately 58 at. $\% \mathrm{Te}$, the proximity of

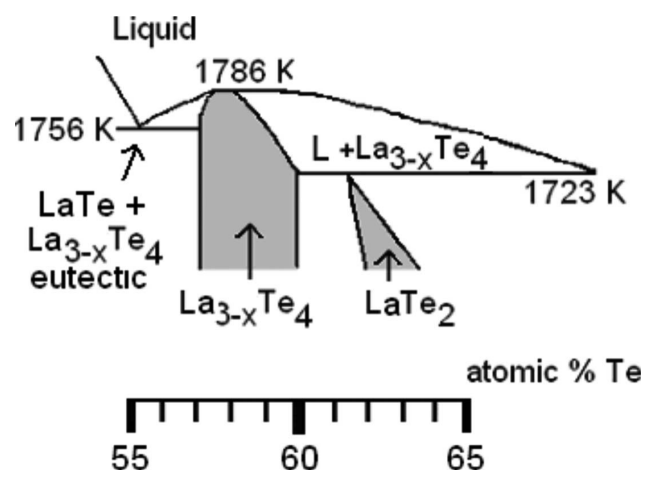

FIG. 2. Lanthanum-tellurium phase diagram for the region of interest (Ref. 13). A solid solution is observed between 57-60 at. \% Te, which corresponds to the $\mathrm{La}_{3-x} \mathrm{Te}_{4}$ phase. High melting points, liquid-solid equilibrium, and a eutectic reaction complicate melt synthesis.

the eutectic reaction necessitates very large quenching rates during solidification and only a small stoichiometric window exists. Together with the high vapor pressure of tellurium, this makes producing high resistance/semi-insulating samples $(x=1 / 3)$ especially difficult.

The many challenges associated with the hightemperature synthesis of $\mathrm{La}_{3-x} \mathrm{Te}_{4}$ highlight the need for a low-temperature synthesis route. To examine what is required for low-temperature synthesis, we consider the kinetics of solid-state reactions. Solid-state reactions are diffusion limited. Therefore the reaction time scales as $t \sim l_{D}^{2} / D_{A}$, where $l_{D}$ is the diffusion length and $D_{A}$ the atomic diffusion coefficient. Traditionally, elevated temperatures are utilized to increase $D_{A}$, which is an exponential function of temperature in solids and becomes very large in the melt.

To avoid high-temperature synthesis while maintaining reasonable reaction times, it is necessary to decrease the diffusion length $l_{D}$. This is accomplished via mechanical alloying, which reduces the particle size of reactants and provides energy to overcome reaction barriers, as well as introduces defects that enhance diffusion. Mechanical alloying was historically utilized to enhance the mechanical properties of materials, and has recently gained popularity as a means to produce difficult-to-synthesize or novel materials, including thermoelectric materials ${ }^{14}$ and other compounds of the $\mathrm{Th}_{3} \mathrm{P}_{4}$ structure type. ${ }^{15}$ In this system, the production of the desired phase near room temperature increases sample homogeneity and reproducibility by two distinct routes: (a) avoiding solidification reduces concerns about the production of inhomogeneous grains, and (b) reducing elemental tellurium to the $\mathrm{Te}^{-2}$ state at low temperature stabilizes tellurium with respect to evaporative losses. Mechanical alloying also allows multiple samples to be produced in a short time period, and is therefore an ideal tool for studying the relationship between thermoelectric properties and composition in the $\mathrm{La}_{3-x} \mathrm{Te}_{4}$ system.

\section{MATERIALS AND METHODS}

\section{A. Synthesis}

Elemental species were obtained from Alfa Aesar. Lanthanum and tellurium chunks with metals basis purities at 
$99.9 \%$ and $99.999 \%$, respectively, were utilized. All preparation steps are performed in an argon dry box. Lanthanum and tellurium are slivered then combined with stainless steel balls in a stainless steel vial, which is placed into a SPEX CertiPrep ${ }^{\circledR} 8000$ series mixer/mill. Milling is completed in less than a day and yields near $95 \%$ are typical. The yield is observed to increase as the composition shifts toward becoming lanthanum deficient. An argon/vacuum hot press is utilized to assist sintering of the powder at temperatures typically in excess of $1300 \mathrm{~K}$, which produces crack-free samples with density higher than $95 \%$ of the theoretical value for $x<0.26$. The lanthanum deficient samples have proved difficult to sinter. The samples with carrier concentrations of approximately $10^{20} \mathrm{~cm}^{-3}$ (labeled below as $n_{H}^{*}$ $\sim 0.03$ ) have only $85 \%$ theoretical density, while those whose properties are presented in Sec. III B 2 have approximately $90 \%$ of theoretical density.

\section{B. Chemical and mechanical characterization}

X-ray diffraction (XRD) and electron microprobe analysis, including wavelength dispersive spectrometry (WDS), are utilized to examine sample homogeneity, purity, and composition. The XRD scans are performed on a Philips X'Pert diffractometer with a $\mathrm{Cu}$ source under a current of 40 $\mathrm{mA}$ with a voltage of $45 \mathrm{kV}$. A JEOL JXA-8200 electron microprobe is utilized in the backscattered electron mode to create compositional contrast images. Images are taken at magnifications of up to $1000 \times$. However, the images taken at $200 \times$ provide the same evidence of single-phase samples with near theoretical density. The instrument is equipped with WDS, which performs chemical analysis on volumes of roughly a cubic micron. During WDS analysis, an electric potential of $15 \mathrm{kV}$ is utilized, $L \alpha \mathrm{x}$ rays are analyzed, a ZAF correction is employed to account for three matrix effects: atomic number (Z), absorption (A), and fluorescence (F), and elemental tellurium and lanthanum phosphate are used as Te and La standards, respectively. A Netzsch STA 449C simultaneously performs thermogravimetry and differential thermal analysis (TG-DTA). The measurement occurs between room temperature and $1375 \mathrm{~K}$ at $20 \mathrm{~K} / \mathrm{min}$, under argon flow with alumina crucible as reference.

Normal and shear ultrasonic measurements are performed at room temperature using input from a Panametrics 5052 pulser/receiver with the filter at $0.03 \mathrm{MHz}$. The response is recorded via a digital oscilloscope, the Tektronic TDS 5054B-NV. The high-resolution mode is employed for the longitudinal speed of sound and an averaging mode (16 wave forms) is utilized for the transverse speed-of-sound measurements. A Netzsch dilatometer (DIL 402C) measures the coefficient of thermal expansion up to $1225 \mathrm{~K}$ in an argon forming gas of $7 \% \mathrm{H}_{2}$ at $2 \mathrm{~K} / \mathrm{min}$.

\section{Electrical and thermal property measurements}

The properties of interest are the Seebeck coefficient $\alpha$, electrical conductivity $\sigma$, and thermal conductivity $\kappa$, all of which are measured under vacuum of less than 5 $\times 10^{-4}$ torr. Hall-effect measurements characterize the Hall mobility and Hall carrier concentration. Hall data are collected using the van der Pauw technique on two separate systems, with magnetic fields of approximately 1 and $2 \mathrm{~T}^{16}$ The Hall data reported are averages of between 2 and 60 room-temperature measurements. The data for the samples with Hall carrier concentrations near $1.2 \times 10^{20} \mathrm{~cm}^{-3}$ averages between room temperature and $750 \mathrm{~K}$. The van der Pauw technique was utilized to determine electrical conductivity up to $1273 \mathrm{~K}$. Electrical conductivity data were collected using pressure contacts of molybdenum or tungsten. The Seebeck coefficient is measured up to $1273 \mathrm{~K}$ with $\mathrm{W} / \mathrm{Nb}$ thermocouples and a differential light-pipe system similar to the one described by Wood et al. ${ }^{17}$ Thermal diffusivity and heat capacity are measured using a laser-flash method via the Netzsch LFA 457 with a Pyroceram 9606 standard.

\section{RESULTS AND DISCUSSION}

\section{A. Chemical and mechanical characterization}

Chemical characterization via XRD and electron microprobe analysis indicates that only the $\mathrm{La}_{3-x} \mathrm{Te}_{4}$ phase is synthesized. Further evidence for the synthesis of a single stable phase is provided via thermogravimetry and differential thermal analysis, where no significant weight loss or peaks are observed up to $1375 \mathrm{~K}$. Trace amounts of $\mathrm{La}_{2} \mathrm{O}_{2} \mathrm{Te}$ are detected (via XRD) in some samples after thermoelectric characterization, as demonstrated in Fig. 3(a), where all major peaks can be attributed to $\mathrm{La}_{3-x} \mathrm{Te}_{4}$ and the location of the largest nonoverlapping oxide peak is marked by the "O."

The average crystallite sizes (after hot pressing) are determined to be between 20 and $30 \mathrm{~nm}$ by the method of integral breadths using a Scherrer constant of 0.9. ${ }^{18}$ Equilibrium scaling arguments for a diffusion-limited process can be utilized to determine if these length scales are reasonable for a solidstate synthesis. By employing the scaling shown above, a 25 $\mathrm{nm}$ diffusion length requires atomic diffusion coefficients on the order of $10^{-21} \mathrm{~m}^{2} / \mathrm{s}$ for synthesis (equilibrium) to be completed in less than a day. This is comparable to the selfdiffusion coefficient of lanthanum at $350 \mathrm{~K}$, but is orders of magnitude larger than the self-diffusion coefficient of tellurium. ${ }^{19}$ However, the crystals produced via mechanical alloying typically contain large amounts of defects that enhance diffusion. Additionally, the characteristic diffusion length during synthesis is certain to be smaller than the average sintered crystallite size. Therefore the required diffusion coefficients are smaller than suggested here.

Electron probe microanalysis utilizing backscattered electrons [see Fig. 3(b)] and WDS indicate single-phase samples with the desired control of stoichiometry. Five WDS measurements yield an average of 41.89 at. \% lanthanum with a standard deviation of 0.30 at. \% for the sample shown in Fig. 3(b), which is in fair agreement with the nominal composition of 41.15 at. \% La. The WDS analysis consistently yields higher lanthanum concentration than expected. The deviation is shown in Fig. 4(a), where the WDS lanthanum concentration has been converted to carrier concentration via Eq. (2). The deviation is particularly apparent in the $x$ $<0.04$ samples, where WDS analysis generates greater lan- 

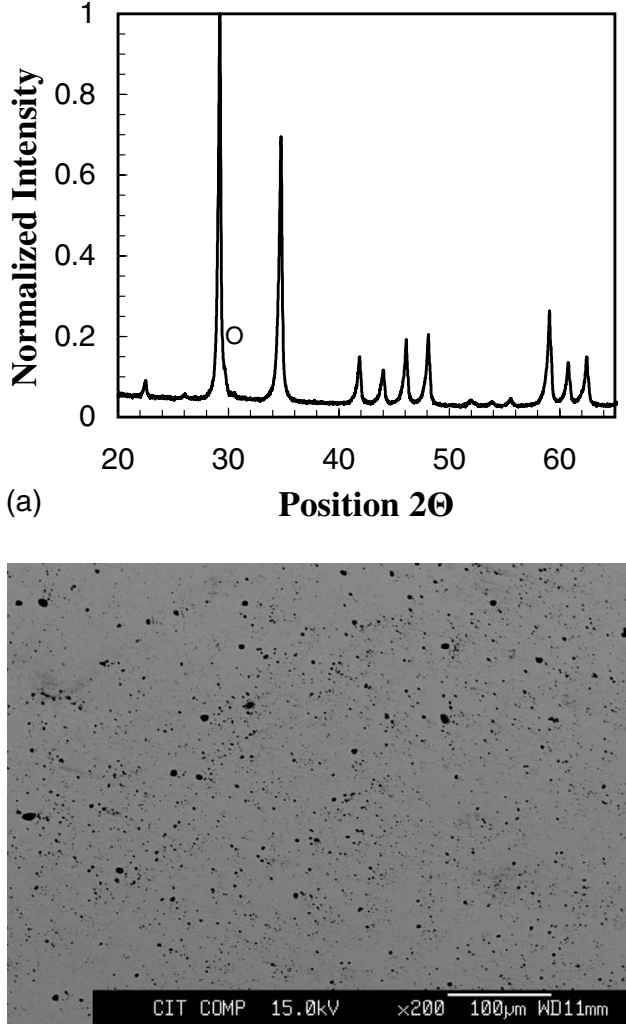

(b)

FIG. 3. Typical XRD scan showing $\mathrm{La}_{3-x} \mathrm{Te}_{4}\left(\mathrm{Th}_{3} \mathrm{P}_{4}\right.$ structure type). This scan was taken after high-temperature thermoelectric characterization that caused minor oxidation. The position of the largest nonoverlapping $\mathrm{La}_{2} \mathrm{O}_{2} \mathrm{Te}$ peak is marked with "O." (b) Backscattered electron image shows a single-phase sample with voids (black circles), and does not indicate presence of the most common impurity, $\mathrm{La}_{2} \mathrm{O}_{2}$ Te. Five WDS measurements on this sample yield an average of 41.9 at. \% $\mathrm{La}$ (41.2 at. \% nominal) with a standard deviation of 0.3 at. $\%$.

thanum concentration than allowed by the crystal structure ( $n>4.5 \times 10^{21} \mathrm{~cm}^{-3}$ or $x<0$ - the dashed line). Therefore, the deviation is attributed mainly to instrumental error, which most likely arises from the standards utilized (elemental Te and $\mathrm{LaPO}_{4}$ ), and the implementation of ZAF matrix correction. Despite this error, the electron microprobe supports the claim of single-phase samples and WDS confirms the composition trend is as desired. One sample contained a small inclusion of iron and chromium, presumably from the milling balls or vial; however, these elements were not found in the $\mathrm{La}_{3-x} \mathrm{Te}_{4}$ matrix.

Knowledge of a material's speed of sound $(v)$ allows an easy characterization of its Debye temperature $\theta_{D}$. Ultrasonic measurements were performed on four samples of nominal composition $\mathrm{La}_{2.81} \mathrm{Te}_{4}$, which resulted in the data shown in Table I, where $\mathrm{M}$ and $\mathrm{C}$ represent the measured and calculated values, respectively. The Debye temperature is calculated to be $208 \mathrm{~K}$ by employing the equation set forth by Anderson, ${ }^{20}$
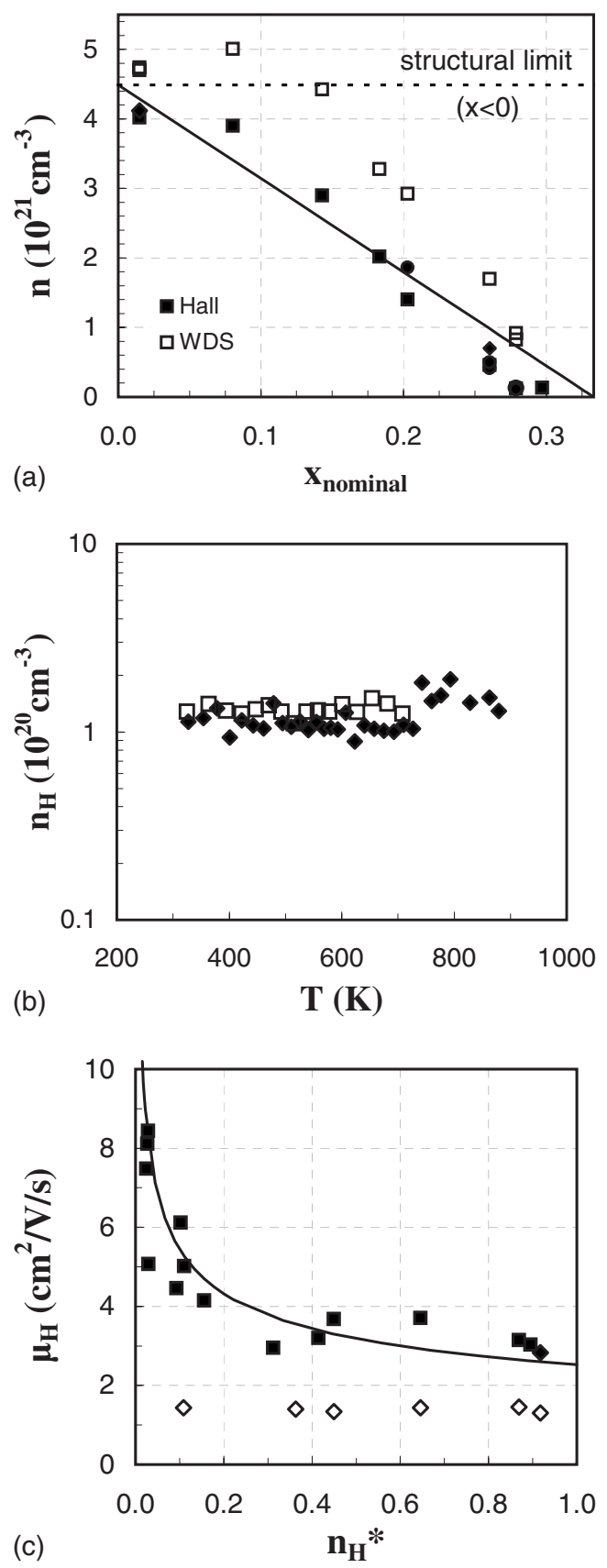

FIG. 4. (a) Hall carrier concentration and WDS measurements presented in terms of carrier concentration demonstrate that carrier concentration changes with nominal composition as desired. The solid curve represents the carrier concentration calculated from nominal composition, and the dashed line represents the maximum carrier concentration allowed by the crystal structure. WDS values larger than the maximum allowed carrier concentration indicate instrumental error. (b) Carrier concentration is found to be constant up to approximately $800 \mathrm{~K}$ for the samples with $n_{H}^{*} \sim 0.03$. (c) Room-temperature Hall mobility decreases with increasing $n ; n_{H}^{*}$ $=n_{H} / n_{\max }$. The solid curve represents $n^{-1 / 3}$ dependence. Roomtemperature data are shown as filled markers and the open diamonds are calculated from conductivity data at $1273 \mathrm{~K}$ and roomtemperature Hall carrier concentration. 
TABLE I. Room-temperature properties for nominal composition $\mathrm{La}_{2.81} \mathrm{Te}_{4}$. $\mathrm{M}$ and $\mathrm{C}$ represent the measured or calculated values, respectively.

\begin{tabular}{lc}
\hline \hline Property & $\begin{array}{c}\text { Measured or } \\
\text { calculated value }\end{array}$ \\
\hline$\alpha_{T}$, coefficient of thermal expansion (M) & $1.5 \times 10^{-5} \mathrm{~K}^{-1}$ \\
$\nu_{\text {long }}$, longitudinal speed of sound (M) & $3580 \mathrm{~m} / \mathrm{s}$ \\
$\nu_{\text {trans }}$, transverse speed of sound (M) & $2010 \mathrm{~m} / \mathrm{s}$ \\
$\Theta_{D}$, Debye temperature (C) & $208 \mathrm{~K}$ \\
$\gamma$, Grüneisen constant (C) & 1.76 \\
$B_{S}$, adiabatic bulk modulus (C) & $5 \times 10^{10} \mathrm{~Pa}$ \\
$\kappa_{l}$, lattice thermal conductivity $(\mathrm{C})$ & $2.0 \mathrm{~W} / \mathrm{m} \mathrm{K}$ \\
\hline \hline
\end{tabular}

$$
\theta_{D}=\frac{h}{k}\left(\frac{3 n N_{A} d}{4 \pi M}\right)^{1 / 3} \nu_{m},
$$

where $n$ is the number of atoms per unit cell, $N_{A}$ is Avogadro's number, $h$ is Planck's constant, $k$ is the Boltzmann constant, $d$ is the density, $M$ is the molecular weight, and $\nu_{m}$ is the appropriately calculated mean speed of sound. This result is consistent with that reported by Tikhonov et al. ${ }^{21}$ who reported a $\Theta_{D}$ of $192 \mathrm{~K}$ at $77 \mathrm{~K}$ for $\mathrm{La}_{2} \mathrm{Te}_{3}$. The thermal-expansion curve was found to be smooth and continuous up to $1225 \mathrm{~K}$, increasing from approximately 15 $\times 10^{-6} \mathrm{~K}^{-1}$ near room temperature to $19 \times 10^{-6} \mathrm{~K}^{-1}$ at high temperature. The coefficient of thermal expansion closely matches that of the $\mathrm{Yb}_{14} \mathrm{MnSb}_{11}$ Zintl compound, which is the best high-temperature $p$-type thermoelectric material. ${ }^{22}$

Using the formulation by Slack and Tsoukala, ${ }^{23}$ the thermal-expansion and speed-of-sound data enable the calculation of additional parameters, including the bulk modulus, Grüneisen constant, and theoretical lattice thermal conductivity. The theoretical lattice thermal conductivity is roughly four times larger than the experimental value, and the large disparity underscores the complexity of this system. The theoretical calculation only accounts for phonon-phonon scattering and does not consider lattice disorder. The large vacancy concentration is not accounted for within the calculation; the presence of vacancies leads to disorder and distortion within the lattice, which is certain to reduce the lattice thermal conductivity. Also, the carrier concentrations examined are relatively high. Thus electron-phonon scattering may play a large role in reducing the lattice thermal conductivity (discussion below).

\section{B. Electrical property measurements and analysis}

\section{Heavily doped semiconductor behavior}

The thermoelectric properties of interest all depend on temperature $T$ and carrier concentration $n$; thus $n$ must be characterized as quantitatively as possible. This is accomplished via the room-temperature Hall effect, which yields a Hall carrier concentration $n_{H}=1 / R_{H} e$, where $R_{H}$ is the Hall coefficient and $e$ is the charge of an electron. Hall carrier concentrations are converted to vacancy parameters $x_{H}$ and the reduced Hall carrier concentration $n_{H}^{*}=n_{H} / n_{\max }$. The Hall factor has been set to unity, which is a good assumption for the small $x$ samples and slightly underestimates the carrier concentration of the larger $x$ samples (for instance, when acoustic phonon scattering dominates, the nondegenerate limit is $\left.r_{H}=1.18\right)$. The room-temperature Hall carrier concentration is shown in Fig. 4(a) as a function of nominal composition, which is characterized by the vacancy parameter $x$. The expected (nominal) carrier concentration calculated by using Eq. (2) is provided. The agreement between the Hall carrier concentration and the nominal one is better than that between the WDS concentration and the nominal one; this demonstrates the need for transport-based characterization of sample composition. The scatter associated with the Hall data can be observed in Fig. 4(b), where the carrier concentration of two samples with $n_{H}^{*} \sim 0.03$ sample is constant up to approximately $750 \mathrm{~K}$, above which the data are too scattered to analyze. For large $n$, the carrier concentration is expected to be constant for all temperatures explored. The Hall carrier concentration and electrical conductivity are utilized to calculate the Hall mobility $\mu_{H}$ from the electrical conductivity,

$$
\sigma=n_{H} e \mu_{H}
$$

The results are shown as a function of $n_{H}^{*}$ in Fig. 4(c). Shown in Fig. 4(c) is the room-temperature Hall mobility (filled markers) and the Hall mobility at $1273 \mathrm{~K}$ calculated utilizing the room-temperature carrier concentration and the data from high-temperature van der Pauw resistivity measurements (open markers).

The room-temperature Hall mobility is found to increase with decreasing $n$. The largest mobility observed is $8.4 \mathrm{~cm}^{2} / \mathrm{V} \mathrm{s}$, for $n_{H}^{*} \sim 0.03$, and the lowest Hall mobility is $3 \mathrm{~cm}^{2} / \mathrm{V} \mathrm{s}$, for $n_{H}^{*}=0.91$. These values are similar to those reported by Danielson et $a .^{24}$ and Vining et al. ${ }^{25}$ for lanthanum telluride, which are slightly larger than the values reported by Cutler et al. ${ }^{3}$ for cerium sulfide, $\mathrm{Ce}_{3-x} \mathrm{~S}_{4}$. At high temperatures, the carrier-concentration dependence of the mobility is lost and the values converge to approximately $1.4 \mathrm{~cm}^{2} / \mathrm{V}$ s. This may suggest that the samples have little degeneracy at $1273 \mathrm{~K}$, because in the limit of a nondegenerate conductor the mobility is predicted to have no carrierconcentration dependence (acoustic phonon scattering of electrons). ${ }^{26}$ However, the $n_{H}^{*}>0.1$ samples have little concentration dependence near room temperature. Samples with low $n_{H}^{*}$ are not included in the high-temperature calculation because the activation of minority carrier cannot be accurately accounted for. The value of $5 \mathrm{~cm}^{2} / \mathrm{V} \mathrm{s}$ at room temperature for the sample with $n_{H}^{*} \sim 0.03$ is lower than expected value due to electron localization or impurity scattering near room temperature, which does not appear to influence the high-temperature conductivity or the temperature dependence of the carrier concentration. Additionally, samples with low $n_{H}^{*}$ contain a large vacancy concentration, which would be expected to decrease carrier mobility, thereby lessening the $n^{-1 / 3}$ dependence.

The behavior demonstrated in Fig. 4(c) suggests that the electron mobility is dominated by acoustic phonon scattering. The solid line shown in Fig. 4(c) represents $n^{-1 / 3}$ depen- 
dence, which is expected for the phonon dominated mobility of "free electrons" experiencing the carrier densities of interest (less than 0.25 charge carriers per atom). In this situation, the mobility can be described as

$$
\mu_{l}=\frac{2^{1 / 3} k h e}{3(2 \pi)^{2}} \frac{\theta_{D}^{2} M}{a m^{* 2} C^{2}} \frac{1}{T n^{1 / 3}},
$$

where $M$ is the average atomic weight, $a$ is the average interatomic distance, $m^{*}$ is the effective mass, $\theta_{D}$ is the Debye temperature found in Table I, and $C$ relates to the phononelectron coupling and is expected to be on the order of 1-10 eV. ${ }^{27}$ The solid line in Fig. 4(c) was calculated using $m^{*}$ $=3.4 m_{e}$, with $M$ corresponding to $x=1 / 6$ and $a=$ the average La-Te bond distance of $3.33 \AA .{ }^{28} C=3.8 \mathrm{eV}$ was selected such that an adequate fit was obtained. The agreement between Eq. (5) and the room-temperature data is surprising because the samples demonstrate a residual resistance and thereby a residual mobility, which is not accounted for in Eq. (5). It is difficult to precisely determine the residual resistances due to the loss of linear temperature dependence in the electrical resistance for samples with $n_{H}^{*}<0.6$. However, a rudimentary analysis reveals that after accounting for the residual resistances, the thermal mobility does increase with decreasing $n$ and roughly follows the $n^{-1 / 3}$ dependence. This trend was not observed by Cutler et al., ${ }^{1}$ who reported that the thermal mobility increased with increasing $n$ in $\mathrm{Ce}_{3-x} \mathrm{~S}_{4}{ }^{1}$

The transport properties of $\mathrm{La}_{3-x} \mathrm{Te}_{4}$ samples display trends typical of heavily doped semiconductors, which are expected when considering samples with $n>10^{20} \mathrm{~cm}^{-3}$. Highly degenerate (metallic) equations often describe the transport data of heavily doped semiconductors quite accurately. This is somewhat surprising because degeneracy in the traditional "metallic" sense is rarely attained. In general, semiconductors are considered degenerate when Boltzmann statistics are no longer a good approximation to Fermi-Dirac statistics, which occurs when the chemical potential $\left(\mu_{F}\right.$, where $\mu_{F}=E_{\mathrm{Fermi}}$ at $0 \mathrm{~K}$ ) begins to leave the band gap and approaches a few $k T$ of the band edge. ${ }^{29}$ In this sense, intrinsic semiconductors are nondegenerate and extrinsic semiconductors are degenerate. However, this guideline requires differentiation between partial (moderate) degeneracy and complete (high) degeneracy. Materials are considered partially degenerate when the reduced chemical potential $(\eta$ $\left.=\mu_{F} / k T\right)$ is near the band edge, in which case Fermi-Dirac statistics are required. Materials are considered highly degenerate when $\eta \gg 0$ (with the zero of energy at the band edge), and calculations can utilize simplifications to the Fermi integrals. ${ }^{26,30}$ Most thermoelectric materials are optimized in the moderately degenerate region. ${ }^{30,31}$

To examine the ability of metallic (highly degenerate) equations to describe this system, we consider the temperature dependence of the Seebeck coefficient. In the case of a highly degenerate conductor, the Seebeck coefficient is expected to increase linearly with temperature and have an $n^{-2 / 3}$ dependence:

$$
\alpha=-\frac{\pi^{2} k^{2} T 8 m^{*}}{3 e h^{2}}\left(\frac{\pi}{3 n}\right)^{2 / 3}(1+\lambda) .
$$

In Eq. (6) $\lambda$ is a scattering constant, which is related to the energy dependence of the electronic scattering distance. ${ }^{1}$ All
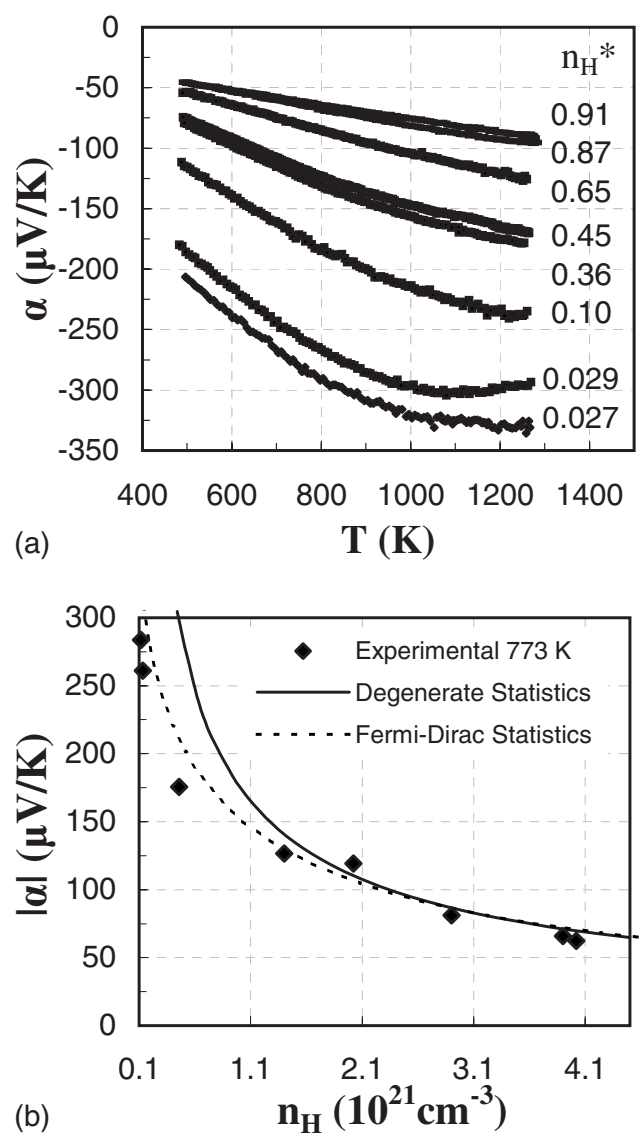

FIG. 5. (a) Seebeck coefficient as a function of temperature with composition indicated by reduced Hall carrier concentration. All samples behave like metallic (degenerate) conductors at low temperatures. Degeneracy is lost at small $n_{H}^{*}$ and high temperatures, and for $n_{H}^{*} \sim 0.03$ samples minority-carrier activation indicates the onset of nondegenerate behavior. (b) Magnitude of Seebeck coefficient vs carrier concentration at $773 \mathrm{~K}$. Curves indicate theoretical calculations: solid line $=$ degenerate statistics and dashed line $=$ Fermi-Dirac statistics.

samples possess degeneracy; the magnitude of the Seebeck coefficient increases linearly with temperature for all samples up to about $900 \mathrm{~K}$, as observed in Fig. 5(a). For large $n$, the samples remain degenerate for all temperatures examined, while for smaller $n$ the samples lose degeneracy and eventually minority carriers are activated. This behavior is easily observed for the $n_{H}^{*} \sim 0.03$ samples, where the Seebeck coefficient initially increases linearly with temperature, then loses linearity, and eventually begins to display a maximum, indicating the thermal activation of minority carriers.

The carrier-concentration dependence of the Seebeck coefficient also provides information regarding degeneracy. To examine the validity of the degenerate assumption, the Seebeck coefficient at $773 \mathrm{~K}$ is plotted versus carrier concentration in Fig. 5(b). The experimental points are compared to two theoretical curves: The solid black line is calculated using Eq. (6). The gray curve represents the more complete calculation using Fermi-Dirac statistics in the relaxation-time approximation solution of Boltzmann transport equations, which takes into account the degree of degeneracy, ${ }^{32}$ 


$$
\alpha=\frac{k}{e}\left\{\frac{(2+\lambda) F_{1+\lambda}}{(1+\lambda) F_{\lambda}}-\eta\right\},
$$

and

$$
n=\frac{4}{\sqrt{\pi}}\left(\frac{2 \pi m^{*} k T}{h^{2}}\right)^{3 / 2} F_{1 / 2}(\eta)
$$

where $F_{r}(\eta)=\int_{0}^{\infty} \xi^{r} f_{0}(\eta) d \xi$, where $f_{0}$ is the Fermi distribution function and $\xi$ is the reduced energy of electrons. The theoretical curves assume a single parabolic band with $m^{*}$ $=3.4 m_{e}$ and $\lambda=0$, which corresponds to acoustic phonon scattering of electrons for the representation shown. The effective-mass value utilized here is consistent with previous results on lanthanum telluride and cerium sulfide. ${ }^{1,24,25}$ For these parameters, it is observed that degenerate statistics should apply for $n$ greater than approximately 2.2 $\times 10^{21} \mathrm{~cm}^{-3}$. Interestingly, all Seebeck coefficient versus temperature curves are increasing linearly with temperature at $773 \mathrm{~K}$. The loss of degeneracy alone does not account for the magnitude of the Seebeck coefficients for $n_{H}^{*}=0.03$ and 0.12. For Eqs. (7) and (8) to describe the Seebeck coefficients of these samples at $773 \mathrm{~K}$, the effective-mass value must be reduced to approximately $2.75 m_{e}$. This implies that the experimental data or the assumption of constant effective mass, scattering constant, and/or single-minimum parabolic bands must be incorrect. Given the complexity of this system and the onset of nondegenerate behavior, we believe the latter to be more probable. However, we recognize that the data have errors in $\alpha$ and $n$ due to measurements and inhomogeneity within a sample. The changes in transport properties corresponding to variations in $n$ are more pronounced for smaller $n$, at which properties change more rapidly. Larger relative errors therefore exist for small $n$.

Another demonstration of the degeneracy of these materials is observed in the temperature and composition dependence of the electrical conductivity; see Fig. 6(a). The electrical conductivity decreases with decreasing $n$ and increasing $T$. This is explained by considering that for a heavily doped large-band-gap semiconductor, $n$ is generally independent of temperature and $\mu$ can be a function of temperature and $n$. In this situation, the direct dependence of $\sigma$ on $n$ is stronger than the dependence of $\mu$ on $n$, and thus $\sigma$ decreases with decreasing $n$. For large degeneracy, the temperature dependence of the mobility is predicted to behave as $T^{-1}$, as shown in Eq. (5). The $T^{-1}$ behavior is observed for large $n$. A transition to the classic $T^{-3 / 2}$ semiconductor behavior is observed as $n$ decreases (when the residual resistance is removed).

The $n_{H}^{*}=0.91$ sample is expected to have a larger conductivity than the $n_{H}^{*}=0.87$ sample. This inconsistency may be the result of many factors, including experimental error and sample inhomogeneity. For the two samples with $n_{H}^{*} \sim 0.03$, the increase in $\sigma$ above $\sim 950 \mathrm{~K}$ indicates the activation of minority carriers, which is consistent with the hightemperature Seebeck coefficient for these samples. The electrical conductivity for the $n_{H}^{*} \sim 0.03$ samples has proven difficult to verify, most likely due to the increased sensitivity to oxidation at low carrier concentration and/or the poor density
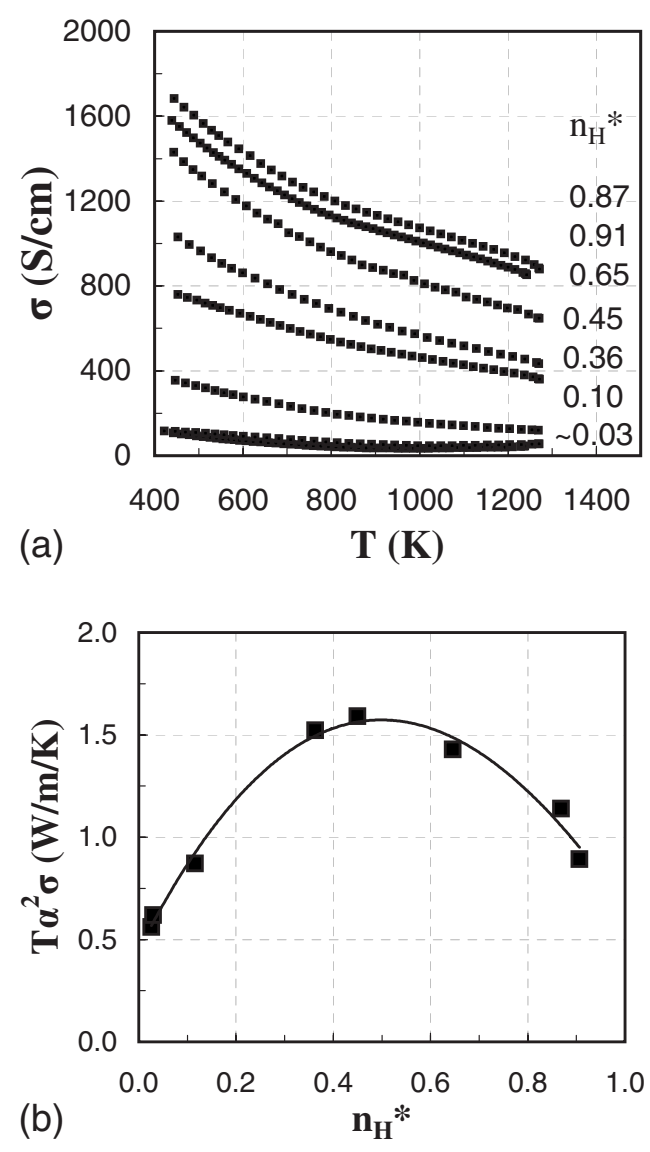

FIG. 6. (a) Electrical conductivity as a function of temperature and composition. Samples with large $n$ have temperature dependence more like that of metals than that of samples with small $n$. Evidence for the thermal activation of minority carriers for two samples with $n_{H}^{*} \sim 0.03$ is observed as $\sigma$ increases slightly above $\sim 950 \mathrm{~K}$. (b) Electrical power factor $\left(T \alpha^{2} \sigma\right)$ at $1273 \mathrm{~K}$ demonstrates the carrier-concentration dependence of a typical semiconductor.

( $\sim 85 \%$ theoretical). A $z T$ curve will be reported for the $n_{H}^{*}$ $=0.029$ sample to demonstrate the magnitude of $z T$ for samples experiencing minority-carrier activation. The irregularity in the curvature of the $z T$ data for $n_{H}^{*} \sim 0.03$ is acknowledged and attributed to the electrical conductivity data.

The last presentation of the electrical transport properties for the high-carrier-concentration samples is the electrical power factor $T \alpha^{2} \sigma$. The power factor at $1273 \mathrm{~K}$ is plotted versus $n_{H}^{*}$ in Fig. 6(b); a maximum value of $1.6 \mathrm{~W} / \mathrm{m} \mathrm{K}$ is obtained near $2 \times 10^{21} \mathrm{~cm}^{-3}$. The compositional dependence of the power factor is very well behaved and follows the expected trend. The guide for the eyes is a polynomial fit and will be utilized below.

\section{Near-insulating behavior}

The insulating limit (stoichiometry, $\mathrm{La}_{2} \mathrm{Te}_{3}$ ) is difficult to obtain, and the transport properties of highly resistive $\mathrm{La}_{3-x} \mathrm{Te}_{4}$ samples have never been reported. The error associated with the electrical resistivity and Seebeck coefficient for these samples is considerably larger than for lowerresistivity samples. This is primarily due to rapid change in 

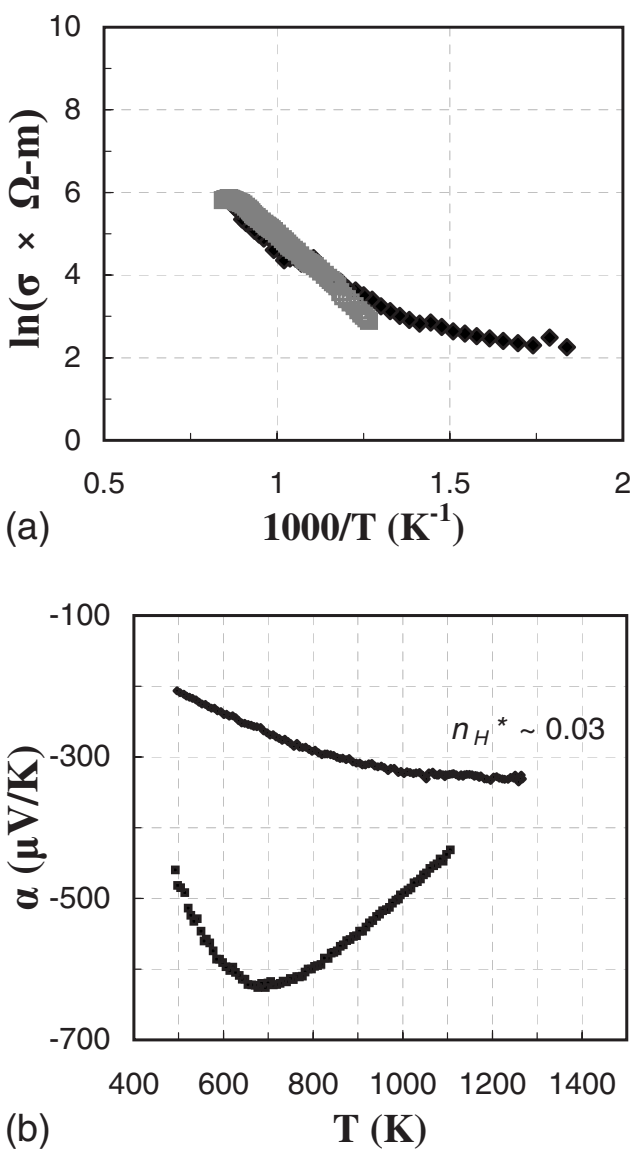

FIG. 7. Electrical properties of near-insulating samples: (a) The natural logarithm of conductivity versus inverse temperature demonstrates the temperature dependence of electrical conductivity for an intrinsic semiconductor at high temperatures. (b) The Seebeck coefficient is very large, dwarfing the Seebeck coefficient of the $n_{H}^{*} \sim 0.03$ sample.

properties at low carrier concentrations and the possibility of inhomogeneity within the sample. Additionally, the low density and increased sensitivity to oxidation makes it difficult to perform multiple measurements on the same sample. As the samples oxidize they experience volumetric and mechanical changes that often result in the loss of electrical contact during van der Pauw measurements. Therefore, the data presented here demonstrate the ability of mechanical alloying to produce near-insulator compositions of $\mathrm{La}_{3-x} \mathrm{Te}_{4}$ while providing information regarding the magnitude of the transport properties and associated parameters for these compositions.

The Hall carrier concentration associated with the electrical conductivity [Fig. 7(a)] and Seebeck coefficient [Fig. $7(b)]$ is known only for the sample represented by black markers in Fig. 7 and was measured to be between $10^{16}$ and $10^{17} \mathrm{~cm}^{-3}$, with considerable scatter. At high temperatures, the electrical conductivity demonstrates the exponential temperature dependence typical of a nondegenerate semiconductor, as shown by the natural logarithmic plot in Fig. 7(a). At moderate temperatures, the small extrinsic carrier concentration causes a deviation from the expected temperature dependence. Assuming that carrier activation is the cause of this temperature dependence, an effective band gap of $0.7 \mathrm{eV}$ $<E_{g}<0.9 \mathrm{eV}$ is calculated for the data shown as black markers, while the gray markers provide $E_{g} \sim 1.3 \mathrm{eV}$. The gray markers represent a second sample of the same nominal composition which was characterized utilizing a four-point technique ${ }^{33}$ on a large cylindrical sample $(\sim 1 \mathrm{~cm}$ in length and $1.2 \mathrm{~cm}$ in diameter), and Hall data are not available. The Seebeck coefficient of the near-insulating sample is, as expected, very large. The maximum Seebeck coefficient allows for an estimate of the band gap via $E_{g}=2 \alpha_{\max } T_{\max } \cdot{ }^{34}$ This analysis yields $E_{g} \sim 0.83 \mathrm{eV}$ for the peak near $670 \mathrm{~K}$, which is consistent with the value obtained for the $n_{H}^{*} \sim 0.03$ sample.

The demonstration of large Seebeck coefficient and nondegenerate electrical conductivity indicates that nearinsulating samples have been produced. The realization of low-carrier-concentration samples is evidence that the main advantage of mechanical alloying is the ability to suppress the vapor phase loss of tellurium. The ability to produce near-insulating samples is a critical step toward the development of a model that accurately describes the metal-insulator transition in $\mathrm{La}_{3-x} \mathrm{Te}_{4}$.

\section{Thermal transport measurements and analysis}

The thermal diffusivity $D_{T}$ is measured by a Netzsch LFA 457 [see Fig. 8(a)]. The instrument also determines heat capacity $C_{p}$ (within approximately $10 \%$ ), allowing the thermal conductivity $\kappa$ to be calculated using the geometric density $d: \kappa=D_{T} C_{p} d$. The heat-capacity data shown in Fig. 8(b) are representative of several samples, and the solid-line fit was utilized to calculate the thermal conductivity of all samples. While the dependence of heat capacity on composition may be of concern, the Dulong-Petit value changes by less than $0.3 \%$ across the stoichiometric window. Thus the actual change in heat capacity with composition is expected to be negligible in comparison to other factors, such as the dependence of the electronic thermal conductivity on carrier concentration. The dashed line in Fig. 8(b) represents the Dulong-Petit (3R) value of the constant volume heat capacity. With a Debye temperature of $208 \mathrm{~K}$, the heat capacity is expected to reach the $3 \mathrm{R}$ value by room temperature and increase with increasing temperature. The $C_{p}$ values reported by Mitarov et al. ${ }^{35}$ for $\mathrm{La}_{3} \mathrm{Te}_{4}$ level off to the Dulong-Petit value at $250 \mathrm{~K}$. It is therefore probable that the $C_{p}$ utilized is an underestimate. At $1275 \mathrm{~K}$, the $C_{p}$ employed here is approximately $20 \%$ larger than the $3 \mathrm{R}$ value.

The thermal conductivity decreases with decreasing $n$ primarily due to the reduction in the electronic thermal conductivity. This is demonstrated in Fig. 9(a), where the total thermal conductivity at $1273 \mathrm{~K}$ is plotted versus $n_{H}^{*}$, and the relation between $n$ and $\kappa_{e}$ indicated by Eqs. (4) and (9) is implied. The data shown in Fig. 9(a) fit fairly well to the solid line, which suggests that a single lattice thermal conductivity $(0.52 \mathrm{~W} / \mathrm{m} \mathrm{K})$ can be utilized to characterize the system. The $\kappa_{L}$ value obtained with this method is low, which certainly contributes to the high $z T$ of this compound. However, changes in the lattice thermal conductivity with carrier concentration must be considered because the va- 

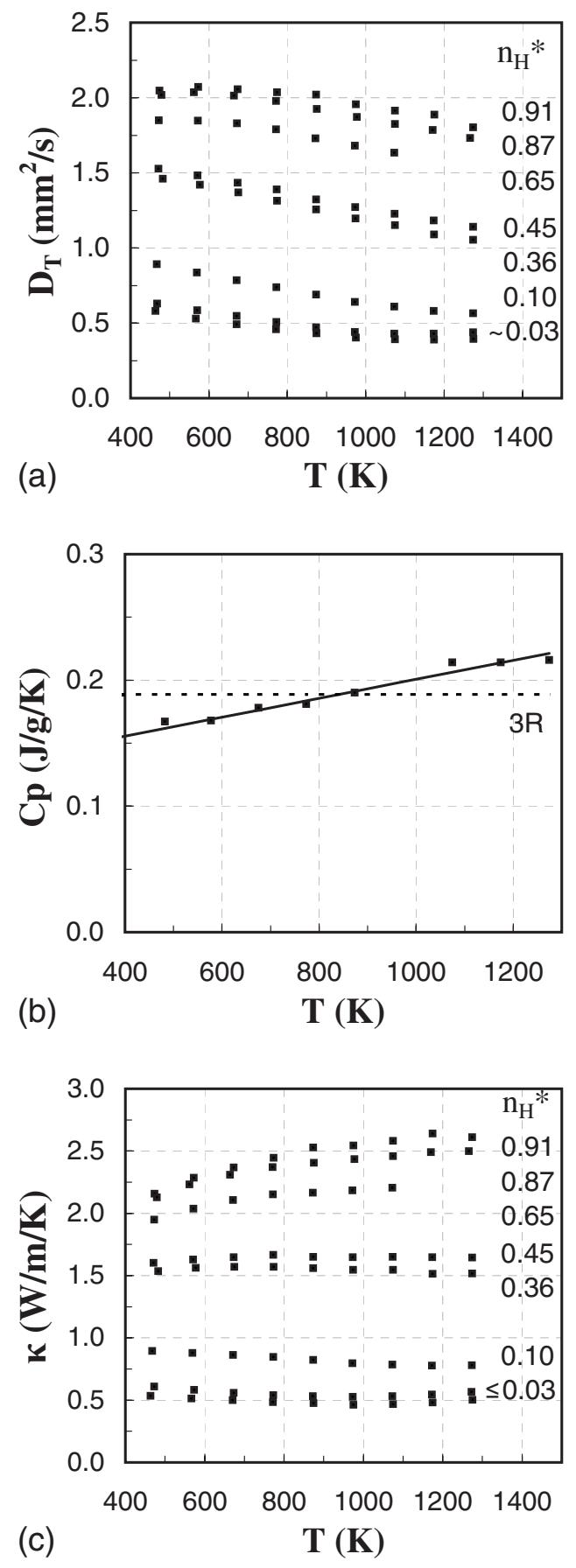

FIG. 8. Thermal transport data: (a) Thermal diffusivity decreases with decreasing $n$ and increasing temperature for all temperatures greater than $600 \mathrm{~K}$. (b) Heat-capacity curve utilized for all samples is compared to the Dulong-Petit (dashed line) value. (c) Thermal conductivity curves show a very large decrease as $n$ decreases due to reduction in electronic thermal conductivity. Samples are identified by their $n_{H}^{*}$ values.

cancy and electron concentrations are coupled. The need for a more rigorous treatment is demonstrated by the dashed line in Fig. 9(a), which is generated by a linear fit to the low- $n$ samples. While the dashed line fits the data for low $n$ well, it yields a lower $\kappa_{L}(0.46 \mathrm{~W} / \mathrm{m} \mathrm{K})$ and predicts higher than
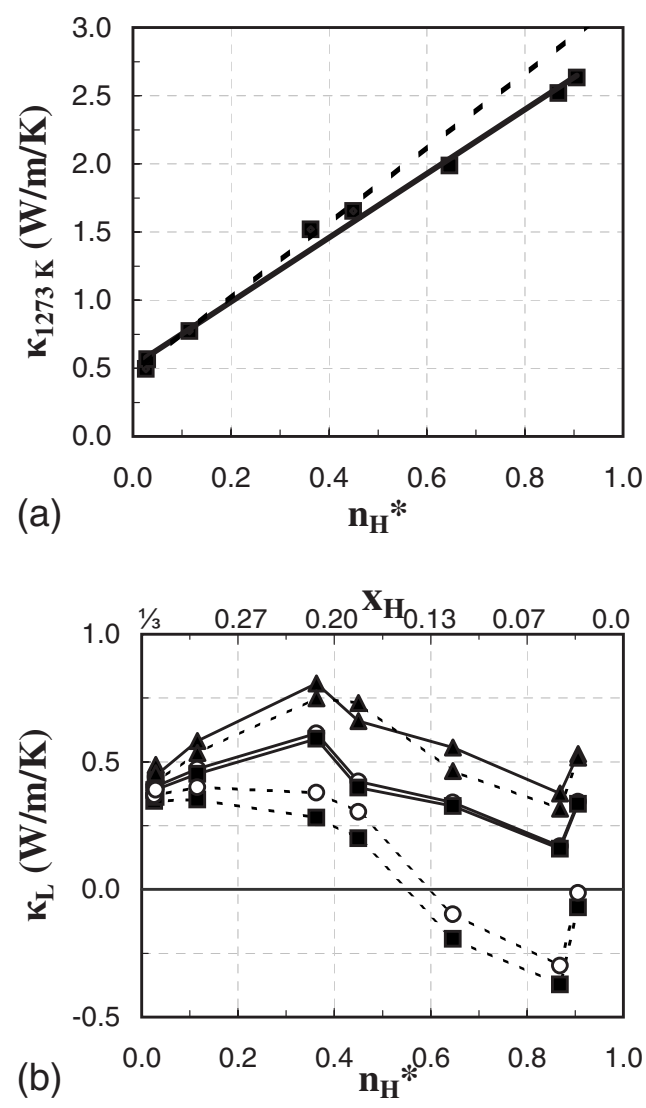

FIG. 9. Investigations of the lattice thermal conductivity via (a) total thermal conductivity vs carrier concentration at $1273 \mathrm{~K}$ and (b) lattice thermal conductivity vs composition for various Lorenz numbers/assumed scattering mechanisms. The apparent maximum near $n_{H}^{*}=0.4$ is attributed to a balance between vacancy/point defect and charge-carrier scattering of phonons. Symbols: $\boldsymbol{\Delta}$-acoustic phonon scattering; $\bigcirc$-free-electron Lorenz values; $\mathbf{\square}$-ionized impurity scattering. Solid lines connect data at $573 \mathrm{~K}$ and dashed lines connect data at $1273 \mathrm{~K}$.

observed thermal conductivity values for the large- $n$ samples.

The lattice thermal conductivity $\kappa_{L}$ is generally calculated by subtracting the electronic portion of thermal conductivity, $\kappa_{e}$, from the total thermal conductivity $\kappa$ by employing the Wiedemann-Franz relationship $\left(\kappa_{e}=L \sigma T\right)$ :

$$
\kappa_{L}=\kappa-\kappa_{e}=\kappa-L \sigma T
$$

To perform this calculation the Lorenz number $L$ is required, and typically the free-electron value of 2.45 $\times 10^{-8} \mathrm{~W} \Omega \mathrm{K}^{-2}$ is assumed. The investigation of the Seebeck coefficient and electrical conductivity indicates that the samples with $n_{H}^{*}>0.03$ have a relatively large degree of degeneracy at moderate temperatures, and one might expect the free-electron $L$ value to be a good approximation. To completely characterize $L$, additional measurements are required to determine the carrier scattering constant $\lambda$. A simpler approach is to assume a value of $\lambda$, determine the reduced chemical potential $(\eta)$ from the measured Seebeck coefficient, and then calculate the Lorenz number. This is accom- 
plished using Eq. (7) and the corresponding equation for the Lorenz number: ${ }^{32}$

$$
L=\left(\frac{k}{e}\right)^{2} \frac{(1+\lambda)(3+\lambda) F_{\lambda} F_{2+\lambda}-(2+\lambda)^{2} F_{1+\lambda}^{2}}{(1+\lambda)^{2} F_{\lambda}^{2}},
$$

where acoustic phonon scattering corresponds to $\lambda=0$ and ionized impurity scattering corresponds to $\lambda=2$.

The lattice thermal conductivity values obtained by utilizing the free-electron Lorenz number and those calculated by using $\lambda=0,2$ are shown in Fig. 9(b) for 575 and $1273 \mathrm{~K}$. The $\kappa_{L}$ values obtained by all assumptions are low, which demonstrates why the rare-earth chalcogenides are attractive for thermoelectric application. For acoustic phonon scattering the Lorenz numbers are between 2.2 and 1.6 $\times 10^{-8} \mathrm{~W} \Omega \mathrm{K}^{-2}$ at $573 \mathrm{~K}$, which yields $\kappa_{L}$ values that are between 0.4 and $0.8 \mathrm{~W} \mathrm{~m}^{-1} \mathrm{~K}^{-1}$. The assumption of ionized impurity scattering yields $L$ values slightly larger than the free-electron value, and thus the value of $\kappa_{L}$ is lower, between approximately 0.2 and $0.4 \mathrm{~W} \mathrm{~m}^{-1} \mathrm{~K}^{-1}$ at $573 \mathrm{~K}$. In the ionized impurity and free-electron limits, $\kappa_{L}$ becomes negative at high temperatures for large $n$, which is due to the large value of $L$ combined with a relatively high value of $\sigma T$. Similarly, all values of $\kappa_{L}$ for small $n$ converge due to the low value of $\sigma T$. This analysis demonstrates that negative $\kappa_{L}$ values do not directly indicate poor measurements but rather highlight the need to use degeneracy-corrected Lorenz numbers when analyzing thermal transport data. Minority-carrier effects are observed in the $n_{H}^{*} \sim 0.03$ samples, which may lead to a lower lattice thermal conductivity if the ambipolar term were taken into account.

The composition dependence of $\kappa_{L}$ can be explained qualitatively by considering phonon-scattering mechanisms. The negative values of $\kappa_{L}$ obtained from the ionized impurity scattering limit provide further evidence that acoustic phonon scattering dominates electron transport. Thus we attempt to explain the maximum in $\kappa_{L}$ near $n_{H}^{*}=0.4$ for the $\lambda=0$ limit. For simplicity, it is assumed that phonon scattering by grain boundaries and impurity atoms is independent of composition. Large- $n$ samples have a low vacancy concentration, and thus the influence of vacancy-derived point-defect scattering is expected to be small. Therefore, the scattering of phonons is most likely dominated by interactions with electrons, which lead to low $\kappa_{L}$ at large $n$. For low $n$, the vacancy concentration is very large and the associated point-defect scattering of phonons significantly reduces $\kappa_{L}$. The balance of these two mechanisms leads to a maximum lattice thermal conductivity near the middle of the stoichiometric range. This somewhat surprising result was also reported by Smirnov et al $^{36}$ as a maximum in $\kappa_{L}$ versus $\sigma$, the location of which is consistent with this study; Smirnov et al. attributed the maximum to an artifact of poor synthesis. The apparent increase in $\kappa_{L}$ at the largest $n_{H}^{*}$ is most likely due to the unexpectedly low electrical conductivity of this sample. Despite the evidence for the dominance of acoustic phonon scattering on the carrier mobility, it is possible that the dominant electron-scattering mechanism changes with composition and the $\kappa_{L}$ versus $n_{H}^{*}$ curves shown are not representative of the system.
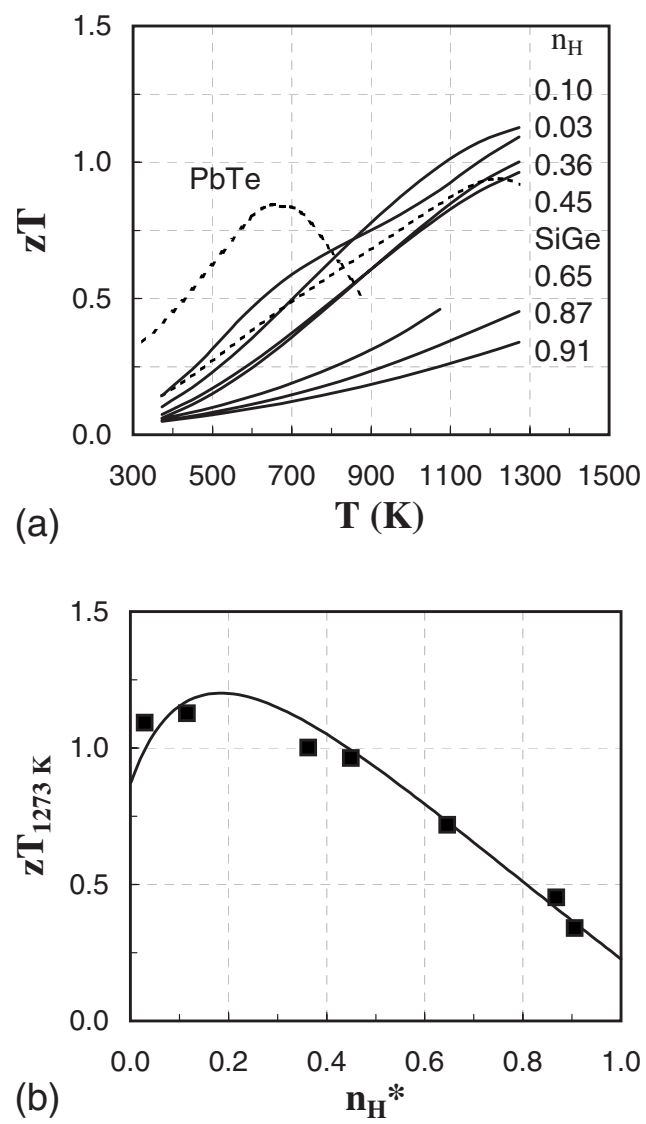

FIG. 10. (a) $z T$ vs temperature for various compositions. The maximum $z T$ is 1.13 at $1273 \mathrm{~K}$ for $n_{H}^{*} \sim 0.03 \quad\left(n_{H} \sim 1.2\right.$ $\times 10^{21} \mathrm{~cm}^{-3}$ ); $z T$ near 1 is obtained for two samples with slightly larger $n$ values. The $z T$ is observed to be larger than that of $n-\mathrm{SiGe}$, which is shown as a dotted line. Also shown are the $n$-PbTe data utilized for segmented-leg efficiency calculations. (b) $z T$ at $1273 \mathrm{~K}$ versus carrier concentration demonstrating expected $n_{H}$ dependence. The solid line was generated using the solid-line fits to the power factor and thermal conductivity.

The exact nature of the electron and phonon scattering in $\mathrm{La}_{3-x} \mathrm{Te}_{4}$ samples is difficult to determine due to the coupling between vacancy and electron concentrations. These two quantities can be decoupled by substituting an element with a different valence for either lanthanum or tellurium, thereby allowing the impact of electron and point-defect scattering of phonons to be more thoroughly investigated.

\section{Figure of merit and thermoelectric compatibility}

The $z T$ values are calculated utilizing the data presented above for $\alpha, \rho$, and $\kappa$. The curves in Fig. 10(a) were generated using polynomial fits to the raw data for $\alpha, \rho$, and the individual components of $\kappa$. No extrapolation is utilized in generating $z T$. Despite the significant variation in properties with composition, several samples have $z T$ near 1 at $1275 \mathrm{~K}$, as shown in Fig. 10(b). A maximum $z T$ of $\sim 1.13$ is obtained for $n_{H}^{*}=0.12$, which is larger than that observed in the SiGe currently employed for deep-space exploration. ${ }^{37}$ Utilizing Eq. (7) we find that the maximum $z T$ values are obtained for 
samples with moderate degeneracy, which is consistent with basic theory.

The guide for the eyes shown in Fig. 10(b) was generated using the fits shown in Figs. 6(b) and 9(a). The quality of these individual fits provides strong support that a $z T$ of 1.2 can be achieved in the pure lanthanum telluride system. Like most thermoelectric materials, the $z T$ is predicted to optimize at a carrier concentration slightly lower than that corresponding to the maximum power factor. ${ }^{38}$ The $z T$ values reported here are similar to those reported by Vining et al., ${ }^{25}$ who reported a maximum $z T$ of approximately 1.2 at $1273 \mathrm{~K}$ for $n / n_{\max }=0.22$, which is consistent with the empirical prediction shown. The samples with $z T$ near $1\left(n_{H}^{*}=0.36,0.45\right)$ correspond to the apparent peak in $\kappa_{L}$. Thus it may be possible to enhance these $z T$ values by introducing point defects at similar carrier concentrations.

In addition to having a relatively high value of $z T$, lanthanum telluride is well suited for a power generation in a segmented device due its thermoelectric compatibility factor $s$, which is defined as

$$
s=\frac{\sqrt{1+z T}-1}{\alpha T} .
$$

The compatibility factor of two adjoining materials must be similar (within a factor of 2) to achieve conversion efficiency in a segmented thermoelectric device near that of an ideal cascaded segmented device. ${ }^{39}$ It is also desirable to have an internally compatible material, which means $s$ is relatively independent of temperature. Lanthanum telluride is observed to be relatively self-compatible at high temperatures; see Fig. 11(a). Also shown in Fig. 11(a) is the compatibility factor of lower-temperature $n$-type materials that would be utilized for segmentation, $\mathrm{CoSb}_{3}$ (Ref. 40) and $\mathrm{PbTe},{ }^{37}$ as well as the current selection for high-temperature application in space exploration, SiGe. ${ }^{37}$ Lanthanum telluride is observed to be more compatible with the lower-temperature materials than SiGe. The influence of the compatibility factor upon device efficiency is demonstrated in Fig. 11(b), where the optimized single-leg efficiencies $\varepsilon_{L}$ for $\mathrm{La}_{3-x} \mathrm{Te}_{4}-\mathrm{PbTe}$ and $\mathrm{SiGe}-\mathrm{PbTe}$ segmented elements are compared. The efficiency is calculated by utilizing an optimization procedure developed previously. ${ }^{39}$ The hot-side temperature was fixed at $1273 \mathrm{~K}$, and the temperature drop across the leg is $600 \mathrm{~K} \leq \Delta T$ $\leq 900 \mathrm{~K}$. The $n_{H}^{*}=0.12$ sample performs more efficiently than SiGe for both segmented and single-material legs for all temperature drops considered. The largest efficiency obtained is approximately $14.3 \%$, which comes from a $\mathrm{La}_{3-x} \mathrm{Te}_{4}-\mathrm{PbTe}$ leg experiencing $\Delta T=900 \mathrm{~K}$. When nonsegmented legs are considered, the $\mathrm{La}_{3-x} \mathrm{Te}_{4}$ sample with the highest $z T$ is more efficient than $\mathrm{SiGe}$ for all temperature drops, with leg efficiencies reaching $11.5 \%$ and $11.2 \%$ for $\Delta T=900 \mathrm{~K}$, respectively.

\section{SUMMARY}

The use of mechanical alloying to produce lanthanum telluride, $\mathrm{La}_{3-x} \mathrm{Te}_{4}$, was demonstrated. The defect-based chemistry of this refractory compound allows the carrier concen-
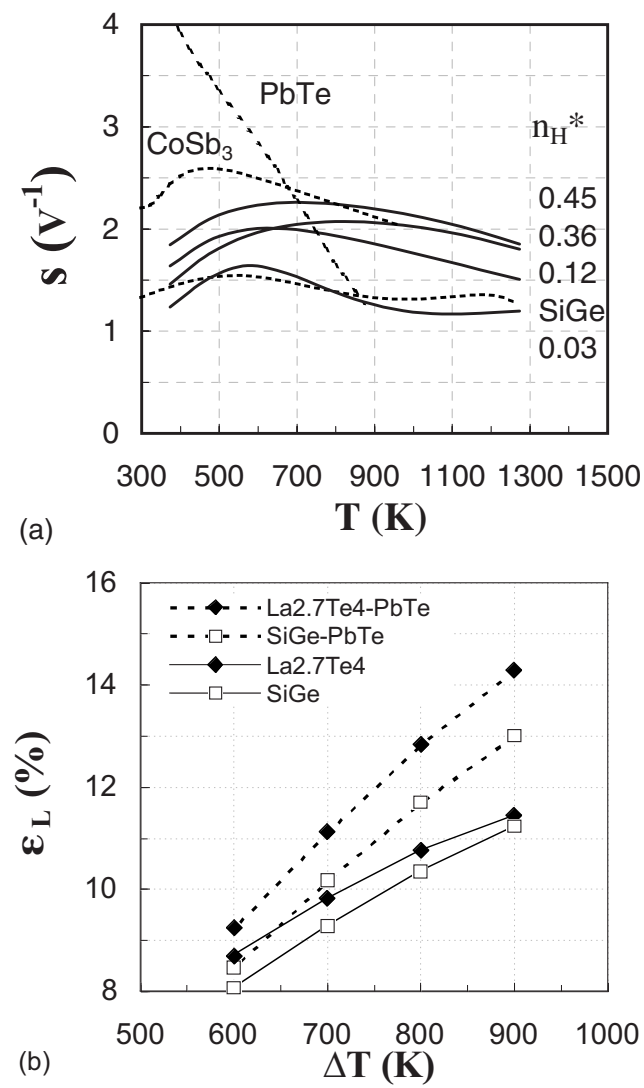

FIG. 11. (a) Thermoelectric compatibility factor $s$ vs temperature for the three high $z T$ samples in this study, as identified by their $n_{H}^{*}$ values, as well as other high $z T$ materials. The compatibility factors of $\mathrm{La}_{3-x} \mathrm{Te}_{4}$ are more favorable than those of $\mathrm{SiGe}$ and are relatively self-compatible (independent of temperature). (b) Leg efficiencies for $\mathrm{La}_{3-x} \mathrm{Te}_{4}-\mathrm{PbTe}$ and $\mathrm{SiGe}-\mathrm{PbTe}$ segmented legs and $\mathrm{La}_{3-x} \mathrm{Te}_{4}$ and $\mathrm{SiGe}$ single-material legs as functions of temperature drop across the leg with a hot-side temperature of $1273 \mathrm{~K}$.

tration and thus the thermoelectric properties to be precisely tuned via stoichiometry control. As expected, the temperature and carrier-concentration dependence typical of heavily doped semiconductors was observed for most samples. Minority-carrier activation and highly resistive nearinsulating behavior were reported, and an effective band gap of approximately $0.9 \mathrm{eV}$ was observed. The synthesis of highly resistive (lanthanum deficient) samples via mechanical alloying emphasizes the significant advantage of mechanical alloying over high-temperature synthesis techniques, which are prone to the vapor phase loss of tellurium that result in higher than desired carrier concentrations.

A maximum $z T$ of 1.13 was obtained (1.2 predicted) at $1273 \mathrm{~K}$ (for Hall carrier concentrations near $5 \times 10^{20} \mathrm{~cm}^{-3}$ and $9 \times 10^{20} \mathrm{~cm}^{-3}$, respectively), which is greater than that observed in $n$-SiGe, the material currently employed in deepspace exploration. A simple single band analysis shows that both carrier-phonon coupling and vacancy disorder are important in producing a low lattice thermal conductivity, which reaches values near $0.4 \mathrm{~W} / \mathrm{m} \mathrm{K}$ and displays a maximum near the middle of the stoichiometric range. The coefficient of thermal expansion was reported to match the p-type complement, the $\mathrm{Yb}_{14} \mathrm{MnSb}_{11}$ Zintl phase. The single- 
leg efficiency calculated for $\mathrm{La}_{3-x} \mathrm{Te}_{4}$ legs is larger than that calculated for state-of-the-art $\mathrm{Si}_{0.8} \mathrm{Ge}_{0.2}$. Furthermore, the thermoelectric compatibility of lanthanum telluride matches the state-of-the-art low-temperature thermoelectric materials significantly better than $\mathrm{SiGe}$, enabling effective segmentation with those materials and efficiency reaches $14.3 \%$ for a temperature drop of $900 \mathrm{~K}$.

\section{ACKNOWLEDGMENTS}

The authors thank the JPL Thermoelectrics Group for help with various measurements and Sossina Haile for useful dis- cussions regarding material processing. They thank Teruyuki Ikeda for performing the electron microprobe measurements and Chi Ma of the Caltech GPS Analytical Facility for discussions regarding the ZAF correction employed. Richard Blair is acknowledged for useful discussions regarding mechanical alloying and crystallite size determination, as are J. Aldrich and Y. Bar-Cohen for providing equipment and assistance with the ultrasonic measurements. The work described in this paper was performed at the Jet Propulsion Laboratory, California Institute of Technology under a contract with the National Aeronautics and Space Administration.
${ }^{1}$ M. Cutler, J. F. Leavy, and R. L. Fitzpatrick, Phys. Rev. 133, A1143 (1964).

${ }^{2}$ M. Cutler and J. F. Leavy, Phys. Rev. 133, A1153 (1964).

${ }^{3}$ M. Cutler, R. L. Fitzpatrick, and J. F. Leavy, J. Phys. Chem. Solids 24, 319 (1963).

${ }^{4}$ V. P. Zhuze, V. M. Sergeeva, and O. A. Golikova, Sov. Phys. Solid State 11, 2071 (1970).

${ }^{5}$ G. G. Gadzhiez, S. M. Ismailov, K. K. Abdullaev, M. M. Khamidov, and Z. M. Omarov, High Temp. 39, 407 (2001).

${ }^{6}$ R. M. Bozorth, F. Holtzberg, and S. Methfessel, Phys. Rev. Lett. 14, 952 (1965).

${ }^{7}$ K. Westerholt, H. Bach, R. Wendemuth, and S. Methfessel, J. Phys. F: Met. Phys. 10, 2459 (1980).

${ }^{8}$ R. N. Shelton, A. R. Moodenbaugh, P. D. Dernier, and B. T. Matthias, Mater. Res. Bull. 10, 1111 (1975).

${ }^{9}$ C. Wood, Rep. Prog. Phys. 51, 459 (1988).

${ }^{10}$ C. Wood, A. Lockwood, J. Parker, A. Zoltan, D. Zoltan, L. R. Danielson, and V. Raag, J. Appl. Phys. 58, 1542 (1985).

${ }^{11}$ L. R. Danielson, V. Raag, and C. Wood, Intersociety Energy Conversion Engineering Conference (Society of Automotive Engineers, Miami, 1985), p. 531.

${ }^{12}$ A. A. Eliseev, E. I. Yarembash, Y. G. Kuznetsov, E. S. Vigileva, A. A. Reshchikova, and L. I. Anotonoa, Russ. J. Inorg. Chem. 9, 482 (1964)

${ }^{13}$ T. H. Ramsey, H. Steinfink, and E. J. Weiss, Inorg. Chem. 4, 1154 (1965).

${ }^{14}$ B. A. Cook and J. L. Harringa, in Thermoelectric Handbook, edited by D. M. Rowe (CRC, Boca Raton, FL, 2006).

${ }^{15}$ T. Ohtani, K. Maruyama, and K. Ohshima, Mater. Res. Bull. 32, 343 (1997).

${ }^{16}$ J. A. McCormack and J. P. Fleurial, Modern Perspectives on Thermoelectrics and Related Materials Symposium (Materials Research Society, Anaheim, CA, 1991), p. 135.

${ }^{17}$ C. Wood, D. Zoltan, and G. Stapfer, Rev. Sci. Instrum. 56, 719 (1985).

${ }^{18}$ H. P. Klug and L. E. Alexander, X-Ray Diffraction Procedures for Polycrystalline and Amorphous Materials (Wiley, New York, 1974).

${ }^{19}$ CRC Handbook of Chemistry and Physics, 2nd ed., edited by R. C. Weast (CRC, Boca Raton, 1981), pp. F54-F59.

${ }^{20}$ O. L. Anderson, J. Phys. Chem. Solids 24, 909 (1963).

${ }^{21}$ V. V. Tikhonov, V. N. Bystrova, R. G. Mitarov, and I. A.
Smirnov, Sov. Phys. Solid State 17, 795 (1975).

${ }^{22}$ A. May, J. Snyder, and J.-P. Fleurial, in Space Technology and Applications International Forum-STAIF 2008, edited by M. S. El-Genk, AIP Conf. Proc. No. 969 (AIP, Melville, NY, 2008), pp. 672-678.

${ }^{23}$ G. A. Slack and V. G. Tsoukala, J. Appl. Phys. 76, 1665 (1994).

${ }^{24}$ L. R. Danielson, M. N. Alexander, C. Vining, R. A. Lockwood, and C. Wood, Proceedings of the Seventh International Conference on Thermoelectric Energy Conversion, Arlington, TX, 1988.

${ }^{25}$ C. Vining, C. Wood, J. Parker, A. Zoltan, L. Danielson, and M. Alexander, Proceedings of the Seventh International Conference on Thermoelectric Energy Conversion, 1988.

${ }^{26}$ V. I. Fistul, Heavily Doped Semiconductors (Plenum, New York, 1969).

${ }^{27}$ A. H. Wilson, The Theory of Metals (Cambridge University Press, Cambridge, England, 1953).

${ }^{28}$ W. L. Cox, H. Steinfink, and W. F. Bradley, Inorg. Chem. 5, 318 (1966).

${ }^{29}$ R. F. Pierret, Advanced Semiconductor Fundamentals (PrenticeHall, Englewood Cliffs, NJ, 2003).

${ }^{30}$ R. R. Heikes and J. R. W. Ure, Thermoelectricity: Science and Engineering (Interscience, New York, 1961).

${ }^{31}$ H. J. Goldsmid, Applications of Thermoelectricity (Wiley, New York, 1960).

${ }^{32}$ I. B. Cadoff and E. Miller, Thermoelectric Materials and Devices (Reinhold, New York, 1960).

${ }^{33}$ C. W. L. D. Zoltan, J.-P. Fleurial, and Y. Liu, Proceedings of the Ninth International Conference on Thermoelectrics, Pasadena, California, 19-21 March 1990, pp. 323-330.

${ }^{34}$ H. J. Goldsmid and J. W. Sharp, J. Electron. Mater. 28, 869 (1999).

${ }^{35}$ R. G. Mitarov, V. V. Tikhonov, L. N. Vasil'ev, V. S. Oskotskii, A. V. Golubkov, and I. A. Smirnov, Sov. Phys. Solid State 17, $310(1975)$

${ }^{36}$ I. A. Smirnov, L. S. Parfen'eva, and V. M. Sergeeva, Sov. Phys. Solid State 14, 899 (1972).

${ }^{37}$ J.-P. Fleurial (private communication).

${ }^{38}$ G. J. Snyder and E. S. Toberer, Nat. Mater. 7, 105 (2008).

${ }^{39}$ G. J. Snyder, in Thermoelectrics Handbook, edited by D. M. Rowe (CRC, Boca Raton, FL, 2006).

${ }^{40}$ G. J. Snyder, Appl. Phys. Lett. 84, 2436 (2004). 